\title{
Effect of different orthotic materials on plantar pressures: a systematic review
}

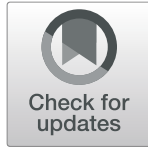

James M. Gerrard ${ }^{1 *}$ (D), Daniel R. Bonanno ${ }^{1,2}$, Glen A. Whittaker ${ }^{1,2}$ and Karl B. Landorf ${ }^{1,2}$

\begin{abstract}
Background: The effect of different orthotic materials on plantar pressures has not been systematically investigated. This study aimed to review and summarise the findings from studies that have evaluated the effect of orthotic materials on plantar pressures.

Methods: We conducted a systematic review of experimental studies that evaluated the effect of foot orthotic materials or shoe insole materials on plantar pressures using in-shoe testing during walking. The following databases were searched: MEDLINE, CINAHL, Embase and SPORTDiscus. Included studies were assessed for methodological quality using a modified Quality Index. Peak pressure, pressure-time integral, maximum force, forcetime integral, contact area, and contact time were variables of interest. Data were synthesised descriptively as studies were not sufficiently homogeneous to conduct meta-analysis. Standardised mean differences (Cohen's d) were calculated to provide the size of the effect between materials found in each study.

Results: Five studies were identified as meeting the eligibility criteria. All five studies were laboratory-based and used a repeated measures design. The quality of the studies varied with scores ranging between 20 and 23 on the modified Quality Index (maximum index score 28). The included studies investigated the effects of polyurethane (including PORON ${ }^{\circledast}$ ), polyethylene (including Plastazote ${ }^{\circledR}$ ), ethyl vinyl acetate (EVA) and carbon graphite on plantar pressures. Polyurethane (including PORON`), polyethylene (including Plastazote ${ }^{\oplus}$ ) and EVA were all found to reduce peak pressure.
\end{abstract}

Conclusion: Based on the limited evidence supplied from the five studies included in this review, some orthotic materials can reduce plantar pressures during walking. Polyurethane (including PORON ${ }^{\circledR}$ ), polyethylene (including Plastazote ${ }^{\circledast}$ ) and EVA reduce peak pressure beneath varying regions of the foot. Future well-designed studies will strengthen this evidence.

Keywords: Orthotic device, Orthosis, Orthoses, Foot, Biomechanics, Kinetics, Plantar pressure

\section{Introduction}

Foot orthoses and shock-absorbing insoles are commonly used to treat and prevent many foot and footrelated conditions [1, 2]. Accordingly, it is imperative that clinicians prescribing foot orthoses have robust evidence on the effects of orthotic materials to inform clinical decision-making. This is particularly pressing given

\footnotetext{
* Correspondence: j.gerrard@latrobe.edu.au

'Discipline of Podiatry, School of Allied Health, Human Services and Sport, La Trobe University, Melbourne, VIC 3086, Australia

Full list of author information is available at the end of the article
}

the forecast of rapid growth within the foot orthotic industry [3]. Evidence should be based on findings from two sources: (i) well-designed randomised trials that evaluate effectiveness based on patient-based outcomes, and (ii) rigorous laboratory-based studies that assess the effects of materials on key biomechanical variables that are linked to effectiveness (i.e. surrogate outcomes) [4].

Regarding laboratory-based outcomes, one of the key effects of orthotic materials on the foot is the influence on plantar pressures [5-7]. Materials can reduce plantar pressure by reducing force or increasing contact area, or 
both. Therefore, materials can influence plantar pressures via their effects on different variables (e.g. force and contact area), which is likely to be dependent on their hardness, density, thickness and ability to contour to the foot. Early research that assessed the effects of orthotic materials was limited to bench-top testing using engineering instrumentation for mechanical analysis (such as a durometer measuring apparatus to assess hardness [8]) and force plate investigations [9], or a combination of the two. However, 'in-shoe' testing using pressure measuring insoles has now become commonplace to examine the effect of orthotic materials on pressure-related variables during gait.

Ideally, standardised results of such 'in-shoe' plantar pressure testing (i.e. from studies that used the same experimental conditions, so they can be directly compared and data pooled in meta-analyses) should be widely available for commonly used orthotic materials. Such data could be used by clinicians when prescribing foot orthoses for patients. For example, it would be highly worthwhile having valid data that is readily available to clinicians (e.g. freely available online) that presents the plantar pressure reductions that commonly used orthotic materials offer, including different thicknesses of those materials. This would enable clinicians the ability to consult such a source to inform their decision-making regarding orthotic materials for patients. Once robust data is available, guidelines could be developed for the materials that foot orthoses are constructed from, so consistent advice is provided to professions that use foot orthoses.

With the above in mind, it is timely to review and summarise the effects of different foot orthotic materials on plantar pressures. The aim of this study was to conduct a systematic review of the current literature by critically evaluating and summarising relevant studies that have assessed the effects of different foot orthotic materials on in-shoe plantar pressures during walking.

\section{Methods}

\section{Search strategy}

This systematic review is reported in accordance with the Preferred Reporting Items for Systematic Reviews and Meta-Analyses (PRISMA) guidelines [10].

The identification of articles for the systematic review was completed with a comprehensive search (Table 1) of titles and abstracts of key electronic databases and additional records. The electronic databases MEDLINE, CINAHL, Embase and SPORTDiscus were systematically searched from inception to March 2020. Broad ranging search terms were agreed on by the authors (JMG, DRB and KBL). The following key scholarly, peer-reviewed journals were also hand searched: Journal of the American Podiatric Medical Association, The Foot, Journal of
Table 1 Search strategy

\begin{tabular}{|c|c|}
\hline & NE and Embase (Ovid) and CINAHL and SPORTDiscus (EBSCO) \\
\hline 1. & exp foot orthosis/ \\
\hline 2. & foot orthoses.mp \\
\hline 3. & $\begin{array}{l}\text { (orthotic* or orthos*s or insole* or heel insert* or ortho* material\$ } \\
\text { or shoe* or footwear or footwear material\$ or sock* or hosier* or } \\
\text { shod).mp }\end{array}$ \\
\hline 4. & 1 OR 2 OR 3 \\
\hline & $\begin{array}{l}\text { (kinetic* or plantar pressure* or peak pressure* or contact area or } \\
\text { contact time or maximum force).mp }\end{array}$ \\
\hline & 4 AND 5 \\
\hline
\end{tabular}

Foot and Ankle Research, and Foot \& Ankle International. Grey literature was searched using: grey literature databases, customised Google search engines, targeted websites and consultation with content experts [11], and recent major conference proceedings were searched using the Web of Science Core Collection database through the La Trobe University Library. All titles and abstracts identified from the search were downloaded to Endnote X8 (Thomson Reuters, Philadelphia, Pennsylvania, USA).

\section{Inclusion and exclusion criteria}

Once all duplicates were removed, the titles and abstracts were independently screened by two authors (JMG and GAW). This process determined whether a study was to be included based on the predetermined eligibility criteria (Table 2), while minimising reviewer bias. The list of selected studies was discussed between

\section{Table 2 Eligibility criteria}

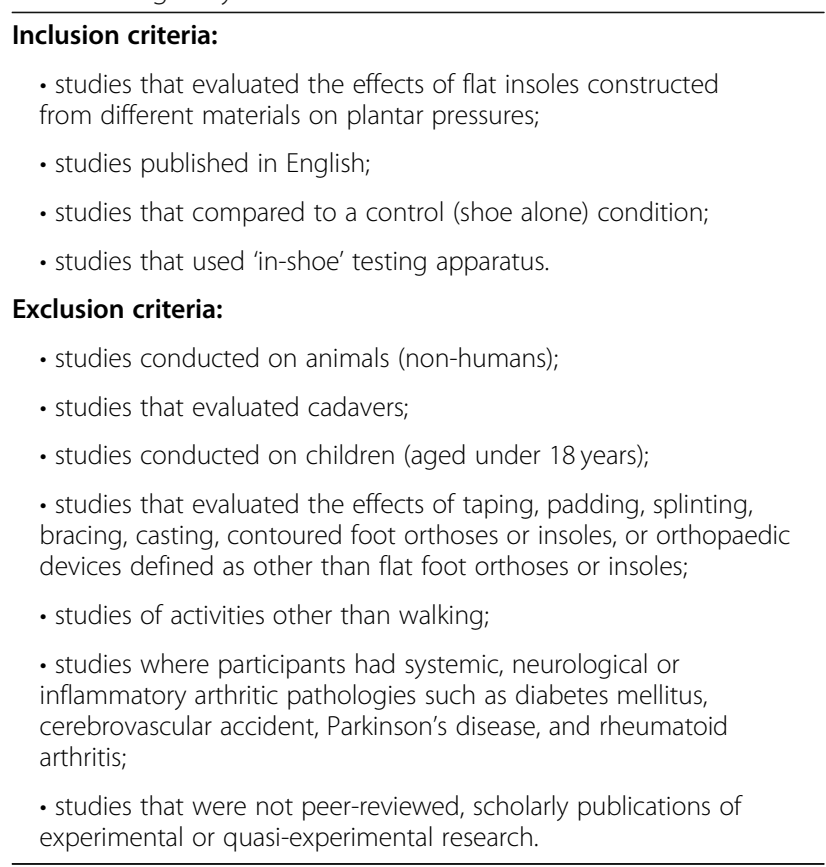


authors until consensus was achieved. If a consensus was not able to be achieved, a third author (DRB) was engaged to officiate any disagreement regarding inclusion/exclusion status of studies. For the purpose of this review, the eligibility criteria included studies that assessed the effects of flat orthotic materials on plantar pressures compared to a control (shoe alone) condition using in-shoe pressure testing while participants who were free from chronic systemic disease were walking. The eligibility criteria were initially applied to all titles and abstracts, and later to full-text articles if more detail was required. All studies that met the inclusion and exclusion criteria, as well as a previous narrative literature review on the topic [5], had their reference lists hand searched for further included articles. In addition, citation tracking was performed using Google Scholar.

\section{Methodological quality assessment}

All studies accepted for review underwent methodological quality assessment using the Quality Index described by Downs \& Black [12]. The Index assesses the quality of non-randomised studies as well as randomised studies [12].

The original Quality Index scale consists of 27 items (maximum score 32) covering four domains: reporting, external validity, internal validity, and power [12]. It has been shown to have high internal consistency (KR-20 $=$ $0.89)$, test-retest $(r=0.88)$ and inter-rater $(r=0.75)$ reliability, and high criterion validity $(r \geq 0.85)$ [12]. In the original Quality Index, criterion 27, which assesses statistical power, is scored between 0 (insufficient statistical power) to 5 (sufficient statistical power). For the purpose of this review, criterion 27 was modified so that studies assessed as having sufficient statistical power (i.e. provided a power calculation) received a score of 1 , and studies that did not provide such detail, or when the statistical power of the study was unable to be determined, received 0. This resulted in the modified Downs and Black Quality Index used in the review maintaining 27 items but having a lower maximum score of 28 compared to the original index. To allow comparability between studies, scores were converted to percentages. Two authors (JMG and DRB), independently scored the included studies. Once all studies were scored, the authors met and discussed any discrepancies until consensus was obtained, at which time a final score was agreed on.

\section{Statistical analysis}

Following methodological assessment, articles were grouped according to materials tested and outcome variables. Data were then synthesised descriptively. Metaanalyses were not conducted because the included studies were not sufficiently homogeneous in terms of participants, interventions, and outcomes. Standardised mean differences (Cohen's $d$ ) [13] were calculated in Microsoft Excel using an appropriate formula [14]. Effect sizes were interpreted as negligible $(0$ to $<0.15)$, small $(0.15$ to $<0.40)$, medium $(0.40$ to $<0.75)$, large $(0.75$ to $<1.10)$, and very large (>1.10) [14].

\section{Results}

The search identified 2332 potential titles and abstracts. Following screening, 19 full-text articles were assessed for eligibility of which 14 were excluded. The remaining five studies were deemed suitable for inclusion (Fig. 1). All the studies used in-shoe analysis to investigate the effect of foot orthotic materials on plantar pressures while walking. Collectively the included studies investigated the following materials: polyurethane (including $\mathrm{PORON}^{\circ}$ ), polyethylene (including Plastazote ${ }^{\circ}$ ), ethyl vinyl acetate (EVA) and carbon graphite. All studies were laboratory-based and used a repeated measures design.

\section{Quality assessment of included studies}

The quality of the five included studies only varied slightly, with Quality Index scores ranging from 20 to 23 (Table 3); the full Downs and Black Quality Index with questions is presented in Additional file 1. All included studies [6, 15-18] clearly stated aims, outcome measures, participant characteristics and interventions. The laboratory-based studies were allocated the maximum score for the criterion that addresses confounders due to their repeated-measures study design. However, no laboratory-based studies satisfied the criterion of reporting adverse events, as such detail is rarely documented in studies of this design involving participants that walked distances of only $10 \mathrm{~m}$ or less in one session lasting no more than several hours. Two laboratory-based studies $[17,18]$ tested participants during a single session, so both were assumed to have satisfied the criterion concerning patients lost to follow-up, as there could not be any.

All of the five studies did report actual probability values (i.e. $p$-values) and all studies were considered as having used appropriate statistical tests to assess the main outcomes data. As recommended in the Quality Index [12], it was assumed that the distribution of data were appropriate for all statistical tests even when not described, such as in the three studies with relatively small sample sizes $(n<11)[6,17,18]$. Of these studies, only Rogers et al. [17] reported that they checked the data were normally distributed and appropriate for parametric testing.

Regarding internal validity, blinding was reported in one of the studies [15]. This study attempted to blind participants to each material being tested, however none of the included studies blinded assessors. Each laboratory-based study did document the interventions 


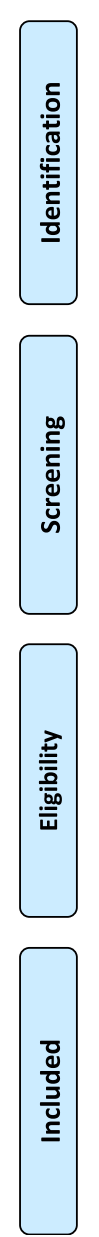

Records identified through database searching in March $2020(n=6941)$ :

CINAHL $(n=1195)$, EMBASE ( $n=$

2650), MEDLINE $(n=1901)$ and

SPORTDiscus ( $n=1195$ )

Additional records identified through

citation tracking and searching

reference lists of previous systematic reviews on related topics $(n=0)$

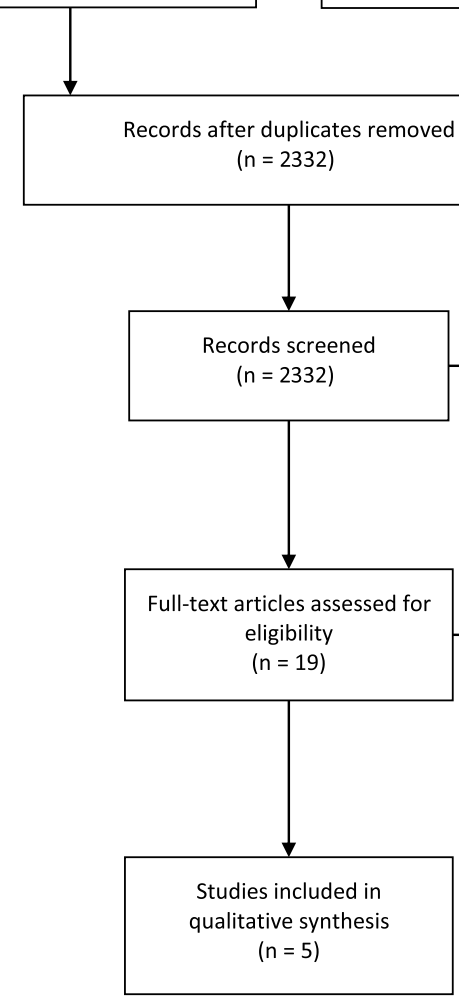

Fig. 1 PRISMA flow diagram

Table 3 Modified Downs and Black Quality Index results for each study

\begin{tabular}{|c|c|c|c|c|c|c|c|c|c|c|c|c|c|c|c|c|c|c|c|c|c|c|c|c|c|c|c|c|c|}
\hline \multirow[t]{2}{*}{$\begin{array}{l}\text { Authors, } \\
\text { date }\end{array}$} & \multicolumn{10}{|c|}{ Reporting } & \multicolumn{3}{|c|}{$\begin{array}{l}\text { External } \\
\text { validity }\end{array}$} & \multicolumn{7}{|c|}{ Internal validity - bias } & \multicolumn{6}{|c|}{$\begin{array}{l}\text { Internal validity - } \\
\text { confounding }\end{array}$} & \multirow{2}{*}{$\begin{array}{l}\text { Power } \\
27\end{array}$} & \multirow[t]{2}{*}{$\begin{array}{l}\text { Total } \\
\text { (max. 28) }\end{array}$} & \multirow[t]{2}{*}{$\begin{array}{l}\text { Total } \\
\%\end{array}$} \\
\hline & 01 & 02 & 03 & 04 & 05 & 06 & 07 & 08 & 09 & 10 & 11 & 12 & 13 & 14 & 15 & 16 & 17 & 18 & 19 & 20 & 21 & 22 & 23 & 24 & 25 & 26 & & & \\
\hline $\begin{array}{l}\text { Healy et al., } \\
2012 \text { [6] }\end{array}$ & 1 & 1 & 1 & 1 & 2 & 1 & 1 & 0 & 0 & 1 & 1 & $U$ & 1 & $U$ & 0 & 1 & 1 & 1 & 1 & 1 & 1 & 1 & 1 & $U$ & 1 & $U$ & 0 & 20 & 71 \\
\hline $\begin{array}{l}\text { McCormick } \\
\text { et al., } 2013 \\
\text { [15] }\end{array}$ & 1 & 1 & 1 & 1 & 2 & 1 & 1 & 0 & 1 & 1 & 1 & $U$ & 1 & 1 & 0 & 1 & 1 & 1 & 1 & 1 & 1 & 1 & 1 & 0 & 1 & 1 & 0 & 23 & 82 \\
\hline $\begin{array}{l}\text { Rao et al., } \\
2009 \text { [16] }\end{array}$ & 1 & 1 & 1 & 1 & 2 & 1 & 1 & 0 & 0 & 1 & 1 & U & 1 & U & U & 1 & 1 & 1 & 1 & 1 & 1 & 1 & 1 & U & 1 & U & 0 & 20 & 71 \\
\hline $\begin{array}{l}\text { Rogers et al., } \\
2006 \text { [17] }\end{array}$ & 1 & 1 & 1 & 1 & 2 & 1 & 1 & 0 & 1 & 1 & 1 & U & 1 & U & 0 & 1 & 1 & 1 & 1 & 1 & 1 & 1 & 0 & 0 & 1 & 1 & 0 & 21 & 75 \\
\hline $\begin{array}{l}\text { Tong \& Ng, } \\
2010[18]\end{array}$ & 1 & 1 & 1 & 1 & 2 & 1 & 1 & 0 & 1 & 1 & 1 & U & 1 & U & 0 & 1 & 1 & 1 & 1 & 1 & 1 & 1 & 0 & 0 & 1 & 1 & 0 & 21 & 75 \\
\hline
\end{tabular}

$0=$ No, $1=$ Yes, $\mathrm{U}=$ Unable to be determined (received a score of 0 )

For the full criteria of the Modified Downs and Black Quality Index see Additional file 1 
for all measuring sessions. Selection bias was minimised in all included studies by having clearly defined recruitment strategies and eligibility criteria. Three laboratorybased studies randomised the interventions that were tested $[6,15,16]$. All studies used valid and reliable inshoe plantar pressure measuring systems; the pedar $-\mathrm{X}^{\circ}$ [19-22] or the F-Scan ${ }^{\text {tix }}[23-26]$.

Regarding external validity (generalisability), no studies specifically reported whether the participants were representative of populations that these orthotic materials would be used on, however all studies provided a setting that was representative of the environment that patients receiving orthotic therapy would experience.

\section{The effect of orthotic materials on plantar pressures}

Of the five studies included in this review, the following materials were investigated: polyurethane (including $\left.\mathrm{PORON}^{\circ}\right)[6,17,18]$, polyethylene (including Plastazote ${ }^{\circ}$ ) [17, 18], ethyl vinyl acetate (EVA) [6, 15] and carbon graphite [16]. In addition to investigating different materials, the studies used a variety of plantar pressure outcome measures and applied a variety of masks to the plantar aspect of the foot, thereby dividing the foot into a range of different anatomical areas. Rao et al. [16] reported major findings only for the medial and lateral midfoot, whereas Rogers et al. [17] reported only the forefoot region, defined as between the base of the hallux and digits and the beginning of the medial arch of the foot. Tong and Ng [18] simply reported both feet as one entity. Healy et al. [6] and McCormick et al. [15] applied similar anatomical masks to the hallux, first metatarsal/1st metatarsophalangeal joint (MTPJ), lateral metatarsals/lateral forefoot, and medial heel and lateral heel. However, Healy et al. [6] combined the midfoot region for analysis, whereas McCormick et al. [15] divided this area into medial and lateral segments.

A summary of the findings from these five studies is presented in Table 4 and Additional file 2.

Polyurethane (an open cell foam) was tested by Healy et al. [6] in both $3 \mathrm{~mm}$ low and medium densities, with both densities being found to reduce peak pressure and peak force across all regions of the plantar foot compared to a control (i.e. shoe alone) condition. The largest reductions in both outcome measures were under the lesser metatarsal region. Low density polyurethane provided a large reduction in peak pressure and peak force (Cohen's $d=0.97$ and Cohen's $d=0.76$, respectively) [6]. Similarly, medium density polyurethane provided a large reduction in both peak pressure and peak force (Cohen's $d=0.96$ and 0.81 , respectively) under the lesser metatarsals [6]. Low density polyurethane also provided a medium reduction in peak pressure (Cohen's $d=0.44$ ) and a small reduction in peak force (Cohen's $d=0.24$ ) at the plantar medial heel [6]. In the same region, medium density polyurethane also provided a medium reduction in both peak pressure and peak force (Cohen's $d=0.44$ and 0.42 , respectively) [6]. In addition, the effect of polyurethane on pressure-time integral was investigated by Healy et al. [6]. Low density polyurethane also provided a medium reduction in the pressure-time integral at the first metatarsal (Cohen's $d=0.44$ ), a large reduction at the lateral metatarsals (Cohen's $d=0.90$ ), and a medium reduction at the medial heel (Cohen's $d=0.61$ ) [6] Medium density polyurethane also reduced pressuretime integral across the same plantar foot regions, providing a medium reduction at the first metatarsal (Cohen's $d=0.54$ ), a medium reduction at the lateral metatarsals (Cohen's $d=0.42$ ), and a small reduction at the medial heel (Cohen's $d=0.32$ ) [6]. Low and medium density polyurethane increased contact area across all plantar regions of the foot [6]. Low density polyurethane provided larger reductions under the hallux, first metatarsal, lesser metatarsals and midfoot plantar regions (Cohen's $d=0.79$ (large), 0.63 (medium), 0.98 (large) and 0.55 (medium), respectively) [6].

Some types of polyurethane are produced and sold under trade names - $P O R O N^{\circ}$ (Rogers Corporation, Chandler, AZ 85224 USA) is one such commonly used insole material. Tong and $\mathrm{Ng}$ [18] found that PORON ${ }^{\circ}$ and slow release $\mathrm{PORON}^{\circ}$ both provided very large reductions in mean peak pressure across the whole foot compared to a control (i.e. shoe alone) condition (Cohen's $d=1.55$ and Cohen's $d=2.01$, respectively). Tong and Ng [18] also found that when adding both a soft and firm Plastazote ${ }^{\circ}$ (Zotefoams plc, Croydon, England) to $P O R O N^{\circ}$, the combination of materials led to very large reductions in peak pressure across the foot compared to a control (i.e. shoe alone) condition (Cohen's $d=1.52$ and Cohen's $d=1.59$, respectively). Similarly, Rogers et al. [17] found that PORON ${ }^{\circ}$ and a combination $\mathrm{PORON}^{\odot} /$ Plastazote $^{\bullet}$ provided very large reductions in peak pressure at the forefoot compared to a control (i.e. shoe alone) condition (Cohen's $d=1.95$ and Cohen's $d=1.70$, respectively). However, with regards to force-time integral at the forefoot, Rogers et al. [17] found that PORON ${ }^{\circ}$ and a PORON $/$ Plastazote $^{\circ}$ combination only provided negligible and small reductions (Cohen's $d=0.14$ and Cohen's $d=0.24$, respectively). The thickness of all $\mathrm{PORON}^{\circ}$, Plastazote and combination PORON ${ }^{\circ} /$ Plastazote $^{\circ}$ insoles tested was $6.2 \mathrm{~mm}$ in the Tong \& $\mathrm{Ng}[18]$ and $6.4 \mathrm{~mm}$ in the Rogers et al. [17] study.

Two studies have investigated EVA $[6,15]$ and they both tested $3 \mathrm{~mm}$ thickness EVA. Healy et al. [6] tested two densities of EVA, which were reported to be 'low density' and 'medium density', while McCormick et al. [15] only tested one density, which was $90 \mathrm{~kg} / \mathrm{m}^{3}$. Regarding peak pressure, Healy et al. [6] found that low 


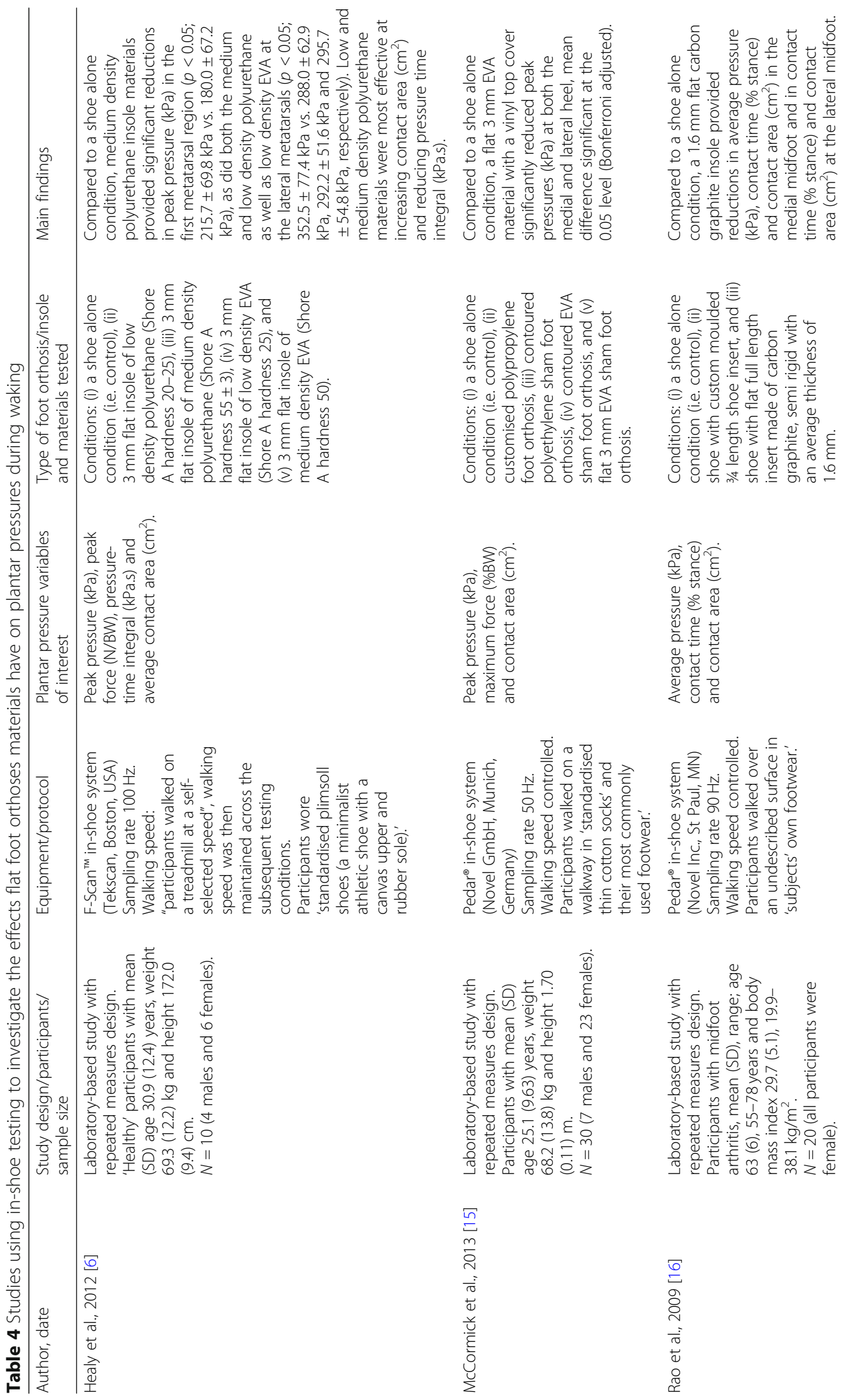




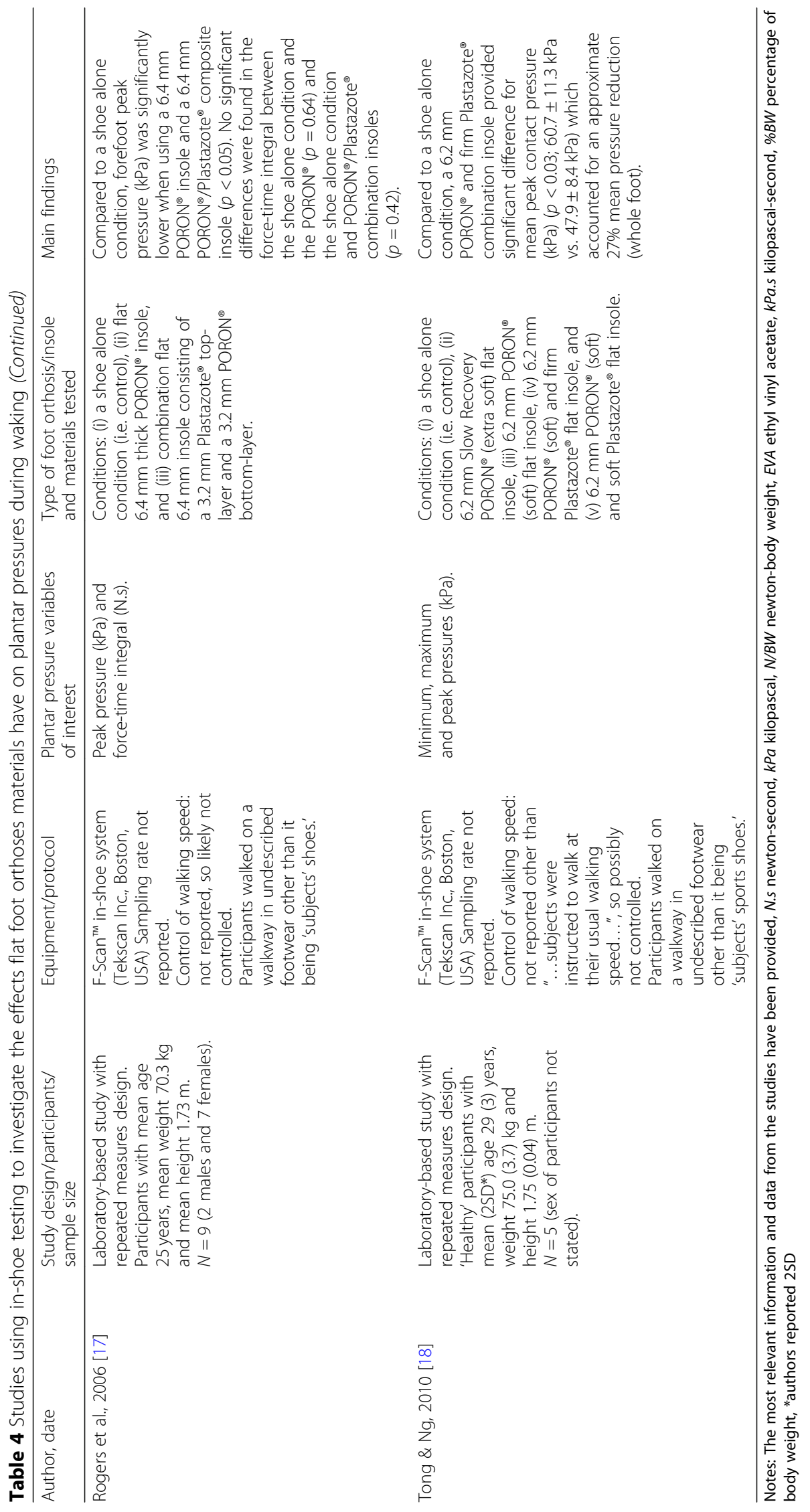


density EVA led to a medium reduction (Cohen's $d=$ 0.46) at the first metatarsal and a large reduction (Cohen's $d=0.89$ ) at the lateral metatarsals compared to a control (i.e. shoe alone) condition. Similarly, McCormick et al. [15] found that $90 \mathrm{~kg} / \mathrm{m}^{3}$ EVA provided a medium reduction of peak pressure (Cohen's $d=0.52$ ) in the medial heel region compared to a control (i.e. shoe alone) condition. Pressure-time integral was also investigated by Healy et al. [6] for low and medium density EVA, but both densities only led to small and negligible effects across all regions of the plantar foot. Regarding contact area, both the low density and the medium density $3 \mathrm{~mm}$ EVA tested by Healy et al. [6] and the $90 \mathrm{~kg} / \mathrm{m}^{3} 3 \mathrm{~mm}$ EVA tested by McCormick et al. [15] increased contact area. The largest increases in contact area were found with low density EVA beneath the medial heel (Cohen's $d=0.30$; small effect) and hallux (Cohen's $d=0.49$; medium effect), with medium density EVA beneath the lateral forefoot (Cohen's $d=0.28$; small effect) and hallux (Cohen's $d=0.43$; medium effect) [6], and with $90 \mathrm{~kg} / \mathrm{m}^{3}$ EVA beneath the medial midfoot (Cohen's $d=0.87$; large effect) and hallux (Cohen's $d=0.61$; medium effect) [15].

Finally, carbon graphite was tested by Rao et al. [16] in $1.6 \mathrm{~mm}$ thickness. In contrast to the other materials that increased contact area under the feet, the use of carbon graphite led to a small reduction in contact area at the forefoot (Cohen's $d=0.33$ ), medium reduction at the midfoot (Cohen's $d=0.47$ ) and small reduction at the heel (Cohen's $d=0.22$ ) compared to a control (i.e. shoe alone) condition [16].

\section{Discussion}

This systematic review has summarised and synthesised the evidence relating to the effect of orthotic materials on plantar pressures while walking shod. All included studies compared orthotic materials in the form of flat insoles to a shoe alone condition by assessing changes in plantar pressures using gold standard in-shoe pressure testing equipment. The methodological quality of the included studies was varied, with McCormick et al. [15] scoring more highly, indicating better internal validity.

Prior to discussing the main findings, several inconsistencies were encountered when reviewing the studies. These inconsistencies are not considered as limitations of the findings of the review, however they do make generalising the findings difficult. These inconsistencies included: variations between studies in thickness of materials tested, most studies omitted standardised durometer hardness values (e.g. Shore values) for the materials tested, and there were differences in masks applied to the plantar surface of the foot for data analysis. Therefore, the conclusions of this review need to be taken into account with reference to the specific thicknesses of material used in each study and that the physical properties of the materials in each study are difficult to compare. In addition, because plantar pressures for different regions of the foot are dependent on mask definition [27], caution is required when comparing data across studies when different masking procedures have been used [28]. A further inconsistency of the included studies was that they did not uniformly report plantar pressure variables. Nevertheless, there is evidence that peak pressure is highly correlated with other plantar pressure variables and some investigators suggest that reporting multiple pressure variables is unnecessary and inefficient $[28,29]$. Despite this, and for the sake of broadening our review, we elected to present all plantar pressure variables reported in the included studies, not just peak pressure. Other inconsistencies between studies include the use of different footwear and walking surfaces (e.g. treadmill versus overground), which may limit external validity and should be considered when interpreting findings.

Inconsistencies aside, polyurethane (including $\mathrm{PORON}^{\circ}$ ) and polyethylene (including Plastazote ${ }^{\circ}$ ) were found to cause substantial changes in plantar pressures $[17,18]$. Polyurethane was found to reduce peak pressure and peak force across all regions of the plantar foot compared to a shoe alone condition [6]. Further, $3 \mathrm{~mm}$ polyurethane provided greater reductions in pressuretime integral than $3 \mathrm{~mm}$ EVA [6]. Both low and medium density polyurethane increased contact area across all plantar regions of the foot [6]. PORON ${ }^{\circ}$ is a specific trade name for a version of polyurethane, and it is commonly used in practice. As $\mathrm{PORON}^{\circ}$ is vulnerable to abrasion, it is most commonly used in combination with a protective cover such as vinyl or cambrelle. When compared to a shoe alone condition, $\mathrm{PORON}^{\circ}$ reduced mean peak pressure [18]. Reducing peak pressure is most often achieved by distributing plantar forces over a larger area via increasing plantar contact area $[9,30]$. In the case of $\mathrm{PORON}^{\circ}$ this occurs due to its relative softness and its ability to conform to the shape of the foot [9]. As a consequence of its plantar pressure reducing capabilities, $\mathrm{PORON}^{\circ}$ is frequently used as an orthotic material [31]. PORON ${ }^{\circ}$ and similar materials may, therefore, be considered to reduce plantar pressures in areas where high pressure is contributing to pain. The following examples highlight some of the conditions that this may be considered: in participants with rheumatoid arthritis, a significant correlation $(r=0.56)$ has been reported between average pressure and pain beneath the second metatarsal head [32]; in participants with pes cavus, a significant correlation has been found between pressure-time integral and foot pain $(r=0.49)$ [33]; and in participants with degenerative foot disorders, a significant correlation $(r=0.52)$ has been reported between average pressure 
beneath the second and third metatarsal heads and pain [34].

$\mathrm{PORON}^{\circ}$ and similar materials may also be indicated for reducing plantar pressures in areas where high pressure can cause tissue break-down, such as with diabetic feet [35]. Raspovic et al. [35] acknowledge that increased plantar pressures often play a significant role in factors leading to tissue breakdown. While it is accepted that sensory neuropathy and ischaemia also contribute to diabetic foot ulceration [36], elevated plantar pressures have been found to contribute the most [37]. A critical threshold pressure value above which ulceration occurs has not yet been identified [38], although mean in-shoe peak pressure at the forefoot of $207 \mathrm{kPa}$ is a suggested target for footwear prescriptions for patients with diabetes with a history of previous foot ulcerations [39]. Nonetheless materials such as $\mathrm{PORON}^{\circ}$ are frequently used in the form of soft orthoses in an attempt to prevent ulcers from forming due to increased plantar pressure [35]. We found evidence in this review to support this practice where studies that evaluated combinations of PORON $^{\circ}$ and Plastazote ${ }^{\circ}$ found reductions in peak pressure $[17,18]$. Plastazote ${ }^{\circ}$ readily moulds to the shape of the foot [40], thus distributing the force applied to the plantar foot over a greater area, so this finding is not unexpected. Likewise, $\mathrm{PORON}^{\circ}$, due to its compressibility, also conforms to the plantar surface of the foot when under load. For this reason, the use of these types of materials in insoles and orthoses [41] is popular among podiatrists, orthotists, and other therapists [18], and is now supported by the evidence we have synthesised in this review.

Like PORON ${ }^{\bullet}$, the effects of EVA on plantar pressures were found to be substantial, particularly under the medial heel and forefoot $[6,15]$. Reduction in peak pressure at the medial heel region is of relevance to plantar heel pain. Reducing pressure under the heel has been suggested to be of benefit for plantar heel pain, particularly when combined with contouring of the orthotic material to produce an orthosis that has a similar shape to the plantar surface of the foot [42]. In addition, reductions at the first and lateral metatarsals hold clinical importance for the treatment of high plantar pressures under the metatarsal heads, for example in the cavus [33] or diabetic foot [35]. Flat EVA (i.e. not moulded) had only a small effect at most on pressure-time integrals across all regions of the plantar foot [6], but this may be clinically important because pressure and time when combined has been postulated to be important in ulcer formation [43-46]. Retrospective studies link high levels of pressure-time integral to plantar ulceration [44], so reducing the combination of pressure and duration that pressure is applied to tissue may be better than reducing peak pressure alone. It is still unknown, however, what reductions in pressure-time integrals are required to reduce the risk of tissue damage/stress related to plantar ulceration [41], but it is plausible that any reduction in pressure-time integral would be advantageous in managing pathology exacerbated by prolonged episodes of sustained pressure. There was no clear consensus for the effect of EVA on maximum force and peak force $[6,15]$ but EVA, particularly in its lower densities, deforms and contours to the shape of the plantar surface of the foot over time, which increases the contact area that the force is applied to, thus reducing plantar pressure.

The other material included in the review was carbon graphite. Carbon graphite is unlikely to be used as an insole material for plantar pressure reduction, however we included it as it was captured in our search. Carbon graphite had the opposite effect on contact area to the other softer materials; that is, it decreased contact area [16]. This is not surprising as the hardness of carbon graphite in the form of an insole decreases the ability of the inner of the shoe, which is often made from softer and more deforming materials, from being able to conform to the plantar foot and thus, increases contact area. Therefore, an insole made from carbon graphite is not a useful material to use if plantar pressure reduction is the sole aim of the insole.

There are eight limitations of this review that need to be acknowledged. First, the majority of the studies included in the review recruited healthy participants who were free from systemic illness, which limits external validity. Because of this, the results cannot be confidently generalised to clinically-relevant populations such as people with diabetic peripheral neuropathy or rheumatoid arthritis, two populations for whom insoles and foot orthoses are commonly prescribed [47-49]. Second, the majority of included studies had small sample sizes, with three studies $[6,17,18]$ having 10 or fewer participants. This may have led to them being underpowered to detect clinically meaningful findings $[50,51]$. Third, two of the studies included in the review $[17,18]$ did not report whether walking speed was controlled for. Walking speed can influence plantar pressure [52], so caution is needed when considering the findings from these two studies. Fourth, comparisons between the pedar- $\mathrm{X}^{\circ}$ and $\mathrm{F}-\mathrm{Scan}^{\mathrm{Ti}}$ are difficult as the technology and specifications vary between systems. For example, the systems use different sensor technologies (pedar is a capacitive-based system, whereas F-Scan is a resistivebased system) [53]. In addition, the systems have different sensor resolutions and data collection frequencies. Fifth, although we calculated effect sizes to provide some clinical meaning to the plantar pressure data reported in the included studies (i.e. from a statistical standpoint), a critical threshold or target for plantar pressure reduction 
that is required to reduce damage (e.g. to prevent ulceration) is still not clear [38]. Sixth, the influence of degradation of the materials due to wear - due to both time and activity - has not been adequately studied, so our review cannot make any conclusions about this issue. Seventh, the review has only considered studies testing orthotic materials in a flat state, as used in a simple insole. Our findings, therefore, do not consider the effect of orthotic materials once they have been altered or manipulated, such as when these materials are heated or added to other materials when manufacturing custommade contoured foot orthoses. Finally, the review excluded non-English language studies, limiting any contribution from such literature.

When considering all of the limitations listed above, and that the materials included in our review have only been tested in one or two studies, our review provides limited evidence that some orthotic materials reduce plantar pressures. This evidence would be strengthened by further well-designed studies that use gold standard in-shoe pressure testing equipment, test materials commonly used in clinical practice, and recruit participants that are representative of clinically-relevant populations where raised plantar pressures have been found to cause problems (e.g. populations that are older, or those that have diabetes or rheumatoid arthritis). In addition, testing the immediate material effects as well as after periods of wear, and attempting to isolate the effects of orthotic materials in flat and contoured states would be worthwhile.

\section{Conclusions}

Based on the limited evidence supplied from the five studies included in this review, some commonly used orthotic materials can reduce plantar pressures during walking. Polyurethane (including PORON ${ }^{\circ}$ ), polyethylene (including Plastazote ${ }^{\circ}$ ), and EVA were found to provide the greatest reductions in plantar pressures. This evidence can be used by clinicians to guide the materials used for insoles.

\section{Supplementary information}

Supplementary information accompanies this paper at https://doi.org/10. 1186/s13047-020-00401-3.

Additional file 1. Full criteria of the Modified Downs and Black Quality Index.

Additional file 2. Detailed results of materials tested with Cohen's d effect sizes to provide comparison between studies.

\section{Abbreviations}

EVA: ethyl vinyl acetate; MTPJ: metatarsophalangeal joint; N.s: newtonsecond; kPa: kilopascal; N/BW: newton-body weight; kPa.s: kilopascal-second; $\%$ BW: percentage of body weight

\section{Acknowledgements}

The authors of this article are employed at La Trobe University and acknowledge the Wurundjeri people of the Kulin Nation as the traditional custodians of the land upon which the Melbourne campus is located. We recognise their ongoing connection to the land and value the unique contribution the Wurundjeri people and all Indigenous Australians make to the university and the wider Australian society.

\section{Authors' contributions}

$J M G, D R B$ and $K B L$ were involved in the preparation and completion of study procedures with GAW participating in the inclusion/exclusion process. $J M G, D R B$ and $K B L$ were responsible for the preparation and review of the manuscript prior to submission for publication. All authors read and approved the final manuscript.

\section{Funding}

Nil sources of funding exist for the research reported. No funding was received for the design of the study or for the collection, analysis, and interpretation of data. There is no funding to declare regarding the writing of this manuscript.

\section{Availability of data and materials}

The datasets used and/or analysed during the current study are available from the corresponding author upon reasonable request.

Ethics approval and consent to participate

Not applicable.

\section{Consent for publication}

Not applicable.

\section{Competing interests}

The authors declare that they have no competing interests.

\section{Author details}

${ }^{1}$ Discipline of Podiatry, School of Allied Health, Human Services and Sport, La Trobe University, Melbourne, VIC 3086, Australia. ${ }^{2}$ La Trobe Sport and Exercise Medicine Research Centre, School of Allied Health, Human Services and Sport, La Trobe University, Melbourne 3086, Australia.

Received: 4 December 2019 Accepted: 22 May 2020

Published online: 11 June 2020

\section{References}

1. Landorf KB, Keenan AM, Rushworth RL. Foot orthosis prescription habits of Australian and New Zealand podiatric physicians. J Am Podiatr Med Assoc. 2001;91(4):174-83.

2. Bonanno DR, Landorf KB, Munteanu SE, Murley GS, Menz HB. Effectiveness of foot orthoses and shock-absorbing insoles for the prevention of injury: a systematic review and meta-analysis. Br J Sports Med. 2017;51(2):86-96.

3. Market Research Store. Foot orthotics insole market (leather, polypropylene and others) by end-user (sports, medical and others): global industry perspective, comprehensive analysis, size, share, growth, segment, trends and forecast, 2015-2021. [http://www.marketresearchstore.com/report/footorthotics-insole-market-z68173] Accessed 10 Jan 2018.

4. Landorf KB. How do foot orthoses work? Podiatry Now. 2016;19(5):24-7.

5. Healy A, Dunning DN, Chockalingam N. Materials used for footwear orthoses: a review. Prosthetics Orthot Int. 2010;2(2):93-110.

6. Healy A, Dunning DN, Chockalingam N. Effect of insole material on lower limb kinematics and plantar pressures during treadmill walking. Prosthetics Orthot Int. 2012;36(1):53-62.

7. Landorf KB, Keenan AM. Efficacy of foot orthoses: what does the literature tell us? J Am Podiatr Med Assoc. 2000;90:149-58.

8. Fauli AC, Andres CL, Rosas NP, Fernandez MJ, Parreno EM, Barcelo CO. Physical evaluation of insole materials used to treat the diabetic foot. J Am Podiatr Med Assoc. 2008;98(3):229-38.

9. Sanfilippo PB, Stess RM, Moss KM. Dynamic plantar pressure analysis. Comparing common insole materials. J Am Podiatr Med Assoc. 1992;82(10): 507-13.

10. Liberati A, Altman DG, Tetzlaff J, Mulrow C, Gotzsche PC, loannidis JP, Clarke M, Devereaux PJ, Kleijnen J, Moher D. The PRISMA statement for reporting 
systematic reviews and meta-analyses of studies that evaluate healthcare interventions: explanation and elaboration. BMJ. 2009;339:b2700.

11. Godin K, Stapleton J, Kirkpatrick SI, Hanning RM, Leatherdale ST. Applying systematic review search methods to the grey literature: a case study examining guidelines for school-based breakfast programs in Canada. Systematic Reviews. 2015:4:138.

12. Downs SH, Black N. The feasibility of creating a checklist for the assessment of the methodological quality both of randomised and non-randomised studies of health care interventions. J Epidemiol Community Health. 1998; 52(6):377-84.

13. Cohen J. A power primer. Psychol Bull. 1992;112(1):155-9.

14. Thalheimer W, Cook S. How to calculate effect sizes from published research articles: a simplified methodology. 2002; [http://www.bwgriffin. com/gsu/courses/edur9131/content/Effect_Sizes_pdf5.pdf. ] Accessed 13 Nov 2019.

15. McCormick CJ, Bonanno DR, Landorf KB. The effect of customised and sham foot orthoses on plantar pressures. J Foot Ankle Res. 2013;6:19.

16. Rao $S$, Baumhauer JF, Becica $L$, Nawoczenski DA. Shoe inserts alter plantar loading and function in patients with midfoot arthritis. J Orthop Sports Phys Ther. 2009;39(7):522-31.

17. Rogers $\mathrm{K}$, Otter $\mathrm{S}$, Birch I. The effect of PORON ${ }^{\circledR}$ and Plastazote ${ }^{\circledR}$ insoles on forefoot plantar pressures. Br J Podiatry. 2006;9(4):111-4.

18. Tong JW, Ng EY. Preliminary investigation on the reduction of plantar loading pressure with different insole materials (SRP-slow recovery Poron ${ }^{\circledR}$ P-Poron ${ }^{\oplus}$, PPF-Poron ${ }^{\oplus}+$ Plastazote, firm and PPS-Poron ${ }^{\oplus}+$ Plastazote, soft). Foot. 2010;20(1):1-6

19. Murphy DF, Beynnon BD, Michelson JD, Vacek PM. Efficacy of plantar loading parameters during gait in terms of reliability, variability, effect of gender and relationship between contact area and plantar pressure. Foot Ankle Int. 2005;26(2):171-9.

20. Putti AB, Arnold GP, Cochrane L, Abboud RJ. The Pedar in-shoe system: repeatability and normal pressure values. Gait Posture. 2007;25(3):401-5.

21. Boyd LA, Bontrager EL, Mulroy SJ, Perry J. The reliability and validity of the novel Pedar system of in-shoe pressure measurement during free ambulation. Gait Posture. 1997;2(5):165.

22. Ramanathan AK, Kiran P, Arnold GP, Wang W, Abboud RJ. Repeatability of the Pedar-X in-shoe pressure measuring system. Foot Ankle Surg. 2010; 16(2):70-3.

23. Woodburn J, Helliwell P. Observations on the F-Scan in-shoe pressure measuring system. Clin Biomech (Bristol, Avon). 1997;12(3):S16.

24. Luo ZP, Berglund $\sqcup$, , An KN. Validation of F-scan pressure sensor system: a technical note. J Rehabil Res Dev. 1998:35(2):186-91.

25. Sumiya T, Suzuki Y, Kasahara T, Ogata H. Sensing stability and dynamic response of the F-scan in-shoe sensing system: a technical note. J Rehabil Res Dev. 1998;35(2):192-200.

26. Nicolopoulos C, Anderson E, Solomonidis S, Giannoudis P. Evaluation of the gait analysis FSCAN pressure system: clinical tool or toy? Foot. 2000;10(3): 124-30.

27. Pataky TC, Caravaggi P, Savage R, Parker D, Goulermas JY, Sellers WI, Crompton $\mathrm{RH}$. New insights into the plantar pressure correlates of walking speed using pedobarographic statistical parametric mapping (pSPM). J Biomech. 2008;41(9):1987-94

28. Keijsers NL, Stolwijk NM, Pataky TC. Linear dependence of peak, mean, and pressure-time integral values in plantar pressure images. Gait Posture. 2010; 31(1):140-2.

29. Waaijman R, Bus SA. The interdependency of peak pressure and pressuretime integral in pressure studies on diabetic footwear: no need to report both parameters. Gait Posture. 2012;35(1):1-5.

30. Foto JG, Birke JA. Evaluation of multidensity orthotic materials used in footwear for patients with diabetes. Foot Ankle Int. 1998;19(12):836-41.

31. Menz HB, Allan JJ, Bonanno DR, Landorf KB, Murley GS. Custom-made foot orthoses: an analysis of prescription characteristics from an Australian commercial orthotic laboratory. J Foot Ankle Res. 2017;10:23.

32. Hodge MC, Bach TM, Carter GM. novel Award First Prize Paper. Orthotic management of plantar pressure and pain in rheumatoid arthritis. Clin Biomech (Bristol, Avon). 1999;14(8):567-75.

33. Burns J, Crosbie J, Hunt A, Ouvrier R. The effect of pes cavus on foot pain and plantar pressure. Clin Biomech (Bristol, Avon). 2005;20(9):877-82.

34. Jannink M, van Dijk H, ljzerman M, Groothuis-Oudshoorn K, Groothoff J, Lankhurst G. Effectiveness of custom-made orthopaedic shoes in the reduction of foot pain and pressure in patients with degenerative disorders of the foot. Foot Ankle Int. 2006;27(11):974-9.

35. Raspovic A, Newcombe L, Lloyd J, Dalton E. Effect of customized insoles on vertical plantar pressures in sites of previous neuropathic ulceration in the diabetic foot. Foot. 2000;10(3):133-8.

36. Reiber GE, Vileikyte L, Ed B, Del Aguila M, Smith DG, Lavery LA, Boulton A. Causal pathways for incident lower-extremity ulcers in patients with diabetes from two settings. Diabetes Care. 1999;22(1):157-62.

37. Frykberg RG, Lavery LA, Pham H, Harvey C, Harkless L, Veves A. Role of neuropathy and high foot pressures in diabetic foot ulceration. Diabetes Care. 1998:21(10):1714-9.

38. Armstrong DG, Peters EJ, Athanasiou KA, Lavery LA. Is there a critical level of plantar foot pressure to identify patients at risk for neuropathic foot ulceration? J Foot Ankle Surg. 1998;37(4):303-7.

39. Owings T, Apelqvist J, Stenström A, Becker M, Bus S, Kalpen A, Ulbrecht J, Cavanagh P. Plantar pressures in diabetic patients with foot ulcers which have remained healed. Diabet Med. 2009;26(11):1141-6.

40. Nicolopoulos C, Black J, Anderson E. Foot orthoses materials. Foot. 2000; 10(1):1-3.

41. Bus SA, Ulbrecht JS, Cavanagh PR. Pressure relief and load redistribution by custom-made insoles in diabetic patients with neuropathy and foot deformity. Clin Biomech (Bristol, Avon). 2004;19(6):629-38.

42. Bonanno DR, Landorf KB, Menz HB. Pressure-relieving properties of various shoe inserts in older people with plantar heel pain. Gait Posture. 2011;33(3): 385-9.

43. Soames R. Foot pressure patterns during gait. J Biomed Eng. 1985;7(2):120-6.

44. Stess RM, Jensen SR, Mirmiran R. The role of dynamic plantar pressures in diabetic foot ulcers. Diabetes Care. 1997;20(5):855-8.

45. Sauseng S, Kästenbauer T, Sokol G, Irsigler K. Estimation of risk for plantar foot ulceration in diabetic patients with neuropathy. Diabetes Nutr Metab. 1999;12(3):189-93.

46. Hsi W-L, Chai H-M, Lai J-S. Comparison of pressure and time parameters in evaluating diabetic footwear. Am J Phys Med Rehabil. 2002;81(11):822-9.

47. Bus SA, Valk G, Van Deursen R, Armstrong DG, Caravaggi C, Hlaváček P, Bakker $K$, Cavanagh $P$. The effectiveness of footwear and offloading interventions to prevent and heal foot ulcers and reduce plantar pressure in diabetes: a systematic review. Diabetes Metab Res Rev. 2008:24(S1):S162-S80.

48. Hennessy K, Woodburn J, Steultjens MP. Custom foot orthoses for rheumatoid arthritis: a systematic review. Arthritis Care Res. 2012:64(3):311-20.

49. Clark H, Rome K, Plant M, O'Hare K, Gray J. A critical review of foot orthoses in the rheumatoid arthritic foot. Rheumatology. 2006;45(2):139-45.

50. Friedman L. Fundamentals of clinical trials. 4th ed. New York: Springer; 2010

51. Faul F, Erdfelder E, Lang A-G, Buchner A. G* power 3: a flexible statistical power analysis program for the social, behavioral, and biomedical sciences. Behav Res Methods. 2007;39(2):175-91.

52. Segal A, Rohr E, Orendurff M, Shofer J, O'Brien M, Sangeorzan B. The effect of walking speed on peak plantar pressure. Foot Ankle Int. 2004;25(12):926-33.

53. Quesada P, Rash S. Quantitative assessment of simultaneous capacitive and resistive plantar pressure measurements during walking. Foot Ankle Int. 2000;21(11):928-34.

\section{Publisher's Note}

Springer Nature remains neutral with regard to jurisdictional claims in published maps and institutional affiliations.

\section{Ready to submit your research? Choose BMC and benefit from:}

- fast, convenient online submission

- thorough peer review by experienced researchers in your field

- rapid publication on acceptance

- support for research data, including large and complex data types

- gold Open Access which fosters wider collaboration and increased citations

- maximum visibility for your research: over $100 \mathrm{M}$ website views per year

At $\mathrm{BMC}$, research is always in progress.

Learn more biomedcentral.com/submissions 